\title{
15. UHURU RESULTS ON EXTRAGALACTIC X-RAY SOURCES
}

\author{
EDWIN M. KELLOGG
}

American Science and Engineering, Cambridge, Mass., U.S.A.

\begin{abstract}
Data from the UHURU satellite have provided a list of more than forty high latitude sources $\left(|b|>20^{\circ}\right)$. X-rays have been detected from among the nearest normal galaxies, giant radio galaxies, Seyferts, QSOs and clusters of galaxies. The cluster sources appear to be extended by several hundred kiloparsecs as well as being very luminous. These cluster sources have systematic differences in their X-ray spectra from individual galaxies.

About twenty sources are not reliably identified so far. A few of these are located near undistinguished $3 \mathrm{C}$ or MSH radio sources. The rest are either located near distant clusters or undistinguished bright galaxies, or are too far south, so that we have not sufficient optical data to allow a thorough search for possible association with clusters or unusual individual galaxies.

The luminosity function for weak, high latitude X-ray sources is determined, and the contribution of sources just below the UHURU threshold of detectability to observed fluctuations in the diffuse $\mathrm{X}$-ray background is evaluated. The total contribution of all observed types of extragalactic sources to the X-ray background is estimated.
\end{abstract}

\section{Introduction}

The UHURU satellite, launched in December 1970, has had a great impact on extragalactic X-ray astronomy. Before data from this satellite became available, there were only a few extragalactic X-ray sources known. Sources near M 87 (Byram et al., 1966 and Bradt et al., 1967) and 3C 273 (Bowyer et al., 1970) and NGC 1275 (Fritz et al., 1971) had been observed definitely, and ones near NGC 5128 (Bowyer et al., 1970), the Coma Cluster (Meekins et al., 1971) and the Large Magellanic Cloud (Mark et al., 1969) had been reported using sounding rocket data. The first fairly complete sky survey with UHURU has revealed more than $40 \mathrm{X}$-ray sources off the galactic plane by $20^{\circ}$ or more. Figure 1 shows the locations in galactic coordinates of the sources

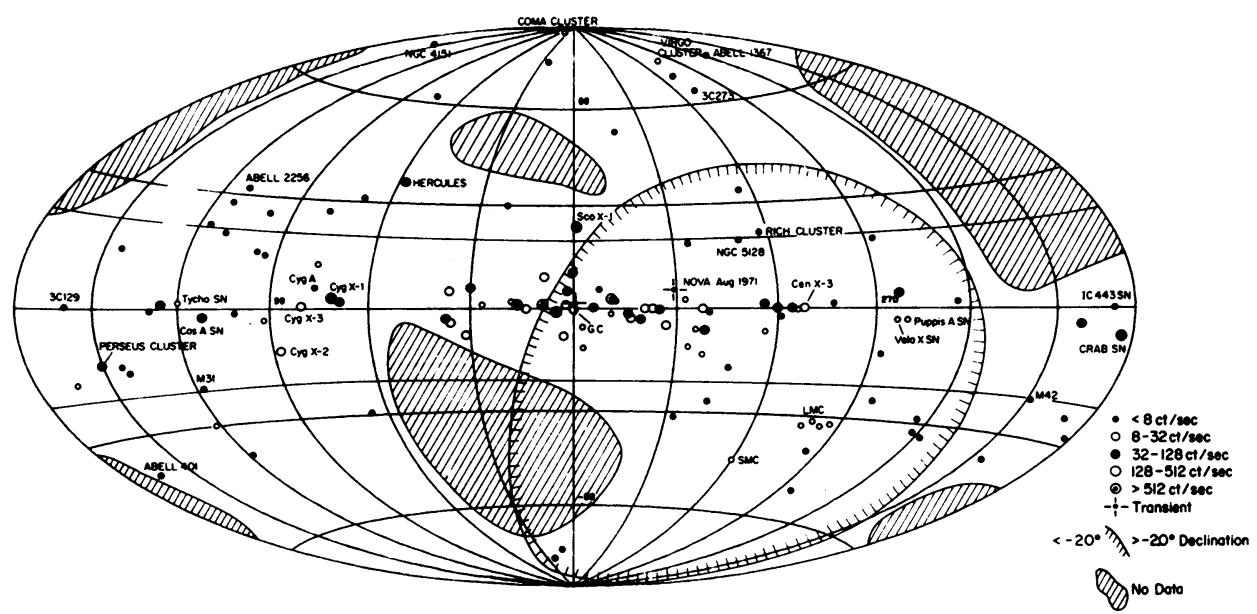

Fig. 1. The X-ray sources in the UHURU Catalog, plotted in galactic coordinates. 
(Giacconi et al., 1972) and it shows some shaded areas we have not yet surveyed. At least fifteen of the high latitude sources are already identified with usual galaxies, or with clusters of galaxies. We believe that most of the remaining unidentified high latitude sources will turn out to be extragalactic.

As was expected, ordinary galaxies are detected as X-ray sources at comparable luminosity to our own galaxy. We also confirm that unusual active galaxies have enhanced X-ray luminosity. The most exciting discovery we have made is that sources in clusters of galaxies are very luminous and are extended, with sizes as large as a few megaparsecs. We also find that the luminosity function of high latitude sources is consistent with a uniform volume distribution as expected for distant extragalactic sources; also that the diffuse background might be the superposition of many distant discrete sources.

\section{Identified Sources}

Figure 2 shows the identified extragalactic sources by class with their distances, ranging from $50 \mathrm{kpc}$ for the Magellanic Clouds to $600 \mathrm{Mpc}$ for the most distant objects. The top of each shaded area represents the distance of the nearest member of each class. The nearest ordinary galaxies are the Magellanic Clouds, located at the top of the shaded area for the class of ordinary galaxies. The Small Cloud is dominated by emission from a single binary X-ray star(Leong et al., 1971 and Schreier et al., 1972.)

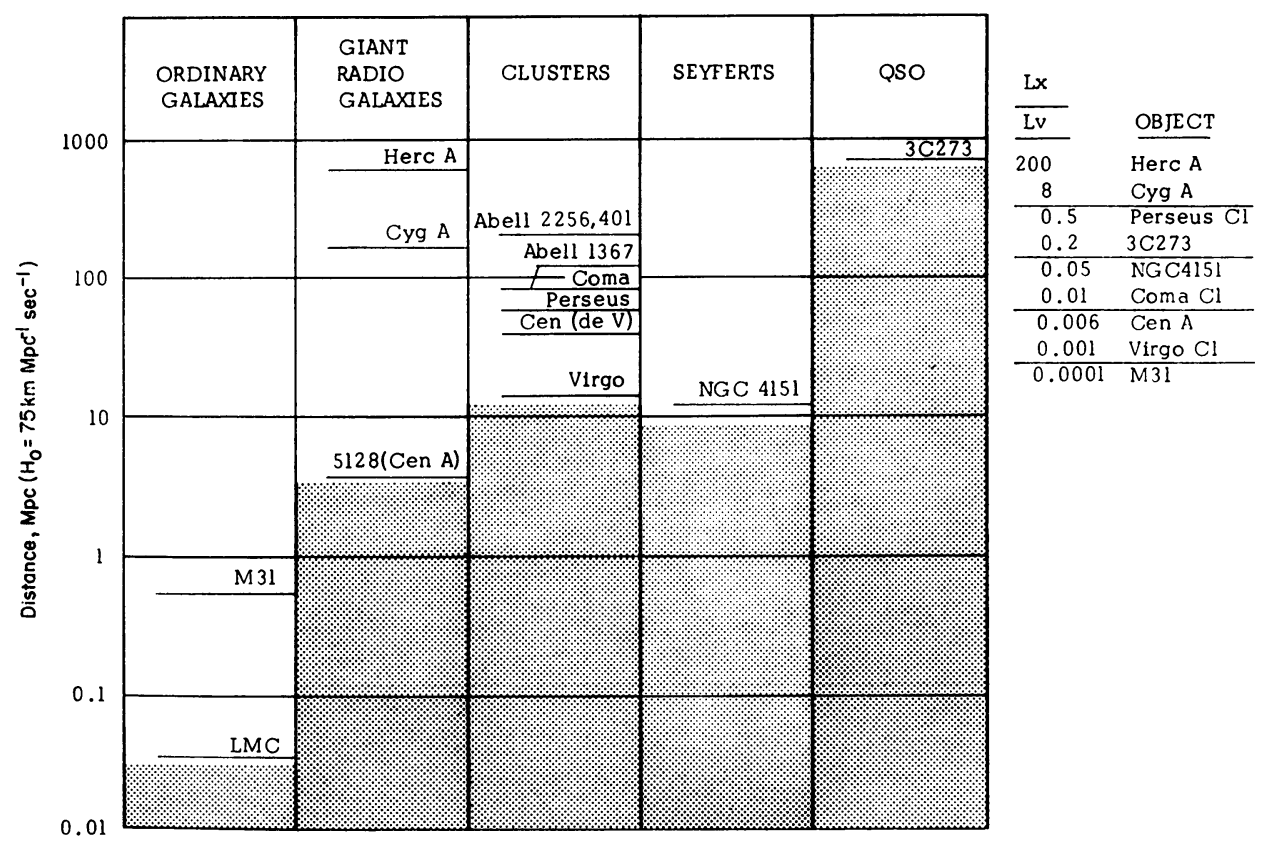

Fig. 2. Identified extragalactic X-ray sources. The top of the shaded region for each class indicates the distance of the nearest known member of that class. The Seyfert galaxies are the only group where the nearest member, NGC 4051, is not detected, but a more distant member is detected. The table at the right lists the ratio of X-1 ay to optical luminosity for representative identified sources. 
The emission from the Large Cloud is due to four discrete sources (Giacconi et al., 1972). The ratio of X-ray to optical luminosity for ordinary galaxies is $10^{-4}$ or less, as listed in the table at the right. We have detected the nearest giant radio galaxy, Cen A (Kellogg et al., 1971 a and Tucker et al., 1972), located at the top of the shaded area for radio galaxies, and two much more luminous distant radio galaxies, Cyg A (Giacconi et al., 1972) and Herc A. Figure 3 shows our best estimates of the locations for Cyg A and Herc A. The updated Herc A location contains more recent data than that included in the UHURU Catalog (Giacconi et al., 1972). The X-ray to visible

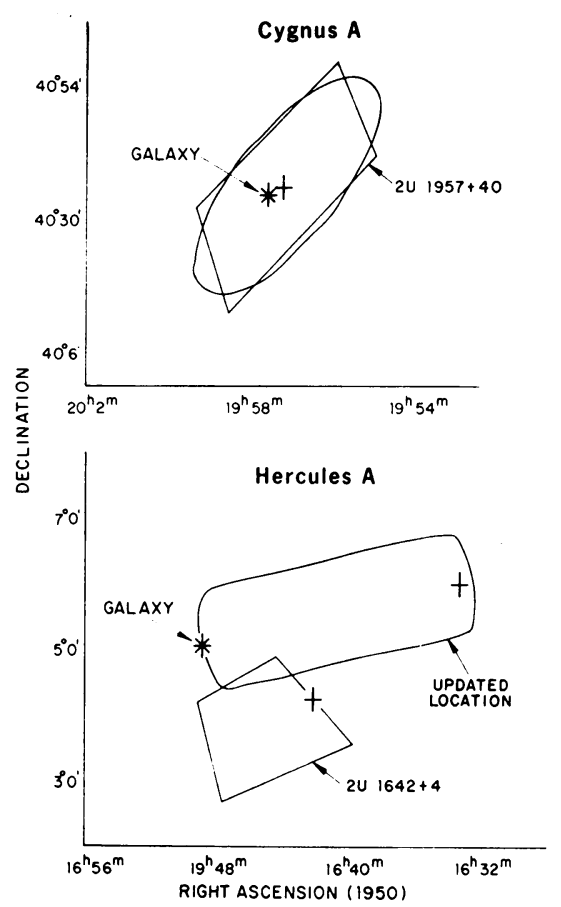

Fig. 3. Locations of two UHURU sources identified with distant radio galaxies. For Cygnus A, the quadrilateral is the error box listed in the UHURU Catalog. The ellipse is a more exact location contour from which the UHURU error box was obtained. The peak of the location probability distribution is shown by a vertical cross. For Hercules A, new data obtained since the UHURU catalog was generated were added to obtain the updated location.

ratios have a large range from $6 \times 10^{-3}$ for Cen A to about 200 for Herc A. However, the X-ray to radio luminosity ratio does not vary so widely for these galaxies. We have detected the nearest great cluster in Virgo, and several more distant rich clusters. Luminosity ratios range from $10^{-3}$ for Virgo to about one in Perseus. We see only one Seyfert galaxy, NGC 4151. The top of the shaded area is at the distance of NGC 4051, the nearest Seyfert, which is not a source. NGC 4051 and NGC 1068 are less than $\frac{1}{3}$ as luminous as 4151 . We also see the nearest QSO, 3C 273. If these classifications are physically meaningful, we'd expect each class to have a typical luminosity; then the 
nearest ones would be detectable first. It appears that only the ordinary galaxies are well behaved in that sense, and possibly QSOs. The other classes show a wide range of luminosities. The enhanced $\mathrm{X}$-ray to visible luminosity ratios for radio galaxies, clusters, the Seyfert and the QSO suggest that the X-rays in these objects are not produced in stars as they are in our own galaxy, but by some other mechanism.

\section{PERSEUS CLUSTER}

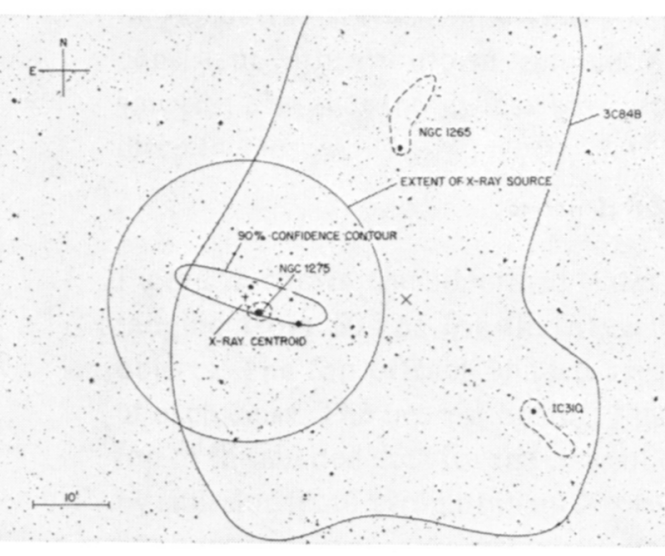

CEN CLUSTER

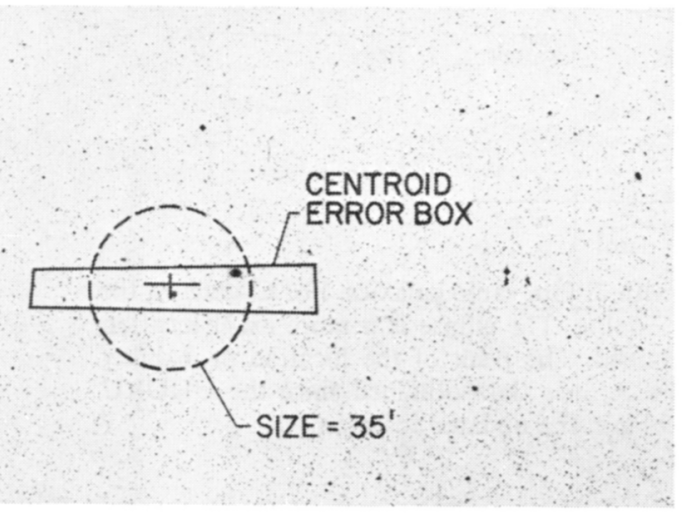

\section{COMA CLUSTER}

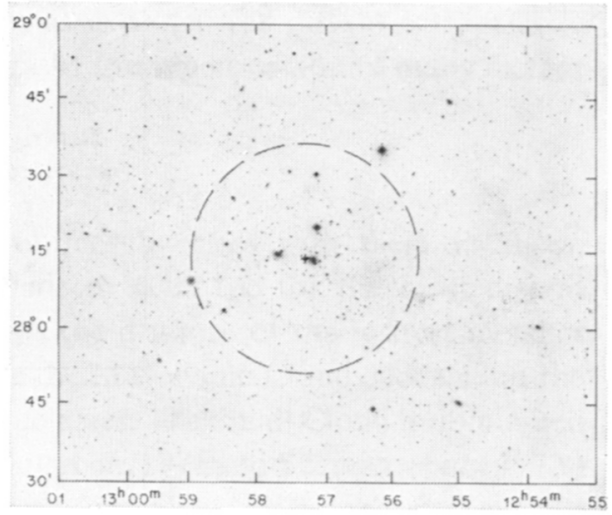

VIRGO CLUSTER

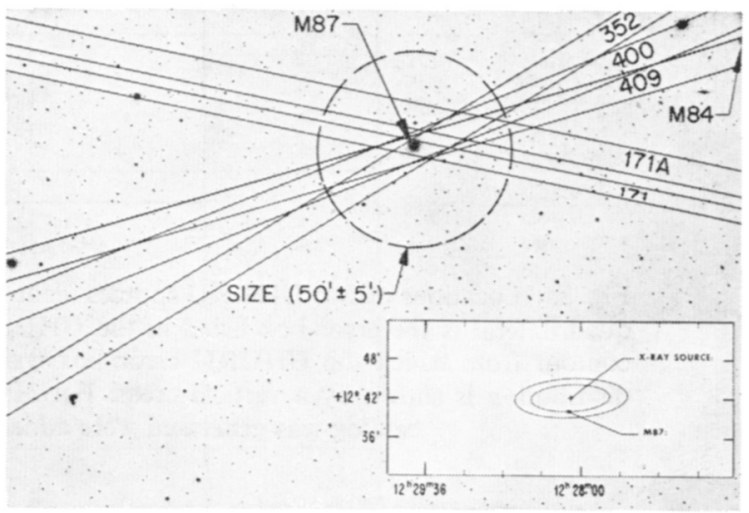

Fig. 4. X-ray sources in clusters of galaxies. These are the four strongest cluster sources. In each case, we indicate the extent of the source by the simplest figure, a circle, since we have no information on the details of the shape. The Virgo picture also shows the one-directional locations for the centroid of the extended source obtained from five scans, labelled by the orbit number pertaining to each scan. The inset is the two-dimensional error box obtained by combining the five one-directional locations. The inner contour corresponds to $68 \%$ confidence and the outer corresponds to $90 \%$ confidence. The Centaurus source, 2U1247-41 contains NGC 4696 within its centroid error box. The Perseus source contains NGC 1275, the exploding Seyfert galaxy, within the centroid error box. In Coma, both the giant elliptical NGC 4874 and the kinematic center of the cluster lie within the error box. (Gursky et al., 1971; Forman et al., 1972; Kellogg et al., 1972). 
The second major finding from UHURU is that sources in clusters are very luminous and extended. We have now identified about 8 sources associated with clusters. In Figure 4, we see four such sources, from the great clusters in Virgo, Cen, Perseus and Coma. The Virgo source is centered on $M$ 87, the active giant elliptical galaxy (Kellogg et al., 1972). The bands are the source centers obtained from 5 different UHURU scans. They intersect at M 87. The inset shows the error box obtained by combining the 5 scans. M 87 is within $3^{\prime}$ of the centroid of the extended X-ray source. Its size is shown by the circle. The true shape of each of these four extended sources is probably not circular, but we cannot tell from our data, so we represent the source shape in the simplest way possible, a circle whose description requires only a single parameter, its diameter.

The Cen cluster is about 3 times further away than Virgo, and the source is weaker. We have just developed a new technique for measuring the size of weaker sources, and we find it to be extended. The giant elliptical galaxy NGC 4696 is located in the error box for the centroid of the extended X-ray source. The size is again shown by a circle, $35^{\prime}$ in diameter. The Perseus cluster source is centered very close to NGC 1275 , a violently active Seyfert galaxy (Forman et al., 1972). The source in Coma may be centered on either the giant elliptical galaxy NGC 4874, or on the kinematic center of the cluster which is very close to 4874 (Forman et al., 1972).

We have tried to measure the angular extent of nine sources located in clusters (see Gursky et al., 1972 for the list of cluster sources). Five of these are definitely extended, as summarized in Figure 5; the other four were too weak to measure sizes. They are Abell 401, Abell 1367, 3C 129, and one Zwicky cluster, and ZW 0444.7+ 0828. The last two sources listed are identified with individual galaxies not located in clusters, and are found not to be extended. Abell 2256 is the most distant extended source we have yet detected and the most luminous. These data show that the clusters have a large range of sizes and luminosities. In fact, there appears to be some correlation between the X-ray luminosity of a cluster and its velocity dispersion, as shown

\begin{tabular}{|c|c|c|c|}
\hline & \multicolumn{2}{|c|}{ SIZE } & \multirow{2}{*}{$\frac{L x}{(\mathrm{erg} / \mathrm{sec})}$} \\
\hline & angular & $k p c$ & \\
\hline ABELL 2256 & $35^{\prime} \pm 15^{\prime}$ & 2800 & $5 \times 10^{44}$ \\
\hline PERSEUS - NGC 1275 & $35^{\prime} \pm 3^{\prime}$ & 740 & $3 \times 10^{44}$ \\
\hline COMA & $36^{\prime} \pm 4^{\prime}$ & 1050 & $2 \times 10^{44}$ \\
\hline CEN - NGC 4696 & $37^{\prime} \pm 8^{\prime}$ & 500 & $2 \times 10^{43}$ \\
\hline VIRGO - M87 & $50^{\prime} \pm 5^{\prime}$ & 200 & $7 \times 10^{42}$ \\
\hline NGC 4151 & $\leq 15^{\prime}$ & $\leq 60$ & $1 \times 10^{42}$ \\
\hline NGC 5128 & $\leq 10^{\prime}$ & $\leq 20$ & $6 \times 10^{41}$ \\
\hline
\end{tabular}

Fig. 5. Sizes and X-ray luminosities of extragalactic X-ray sources. Five extended cluster sources and two compact sources not in clusters are listed. The cluster sources are much more luminous than the compact sources. 


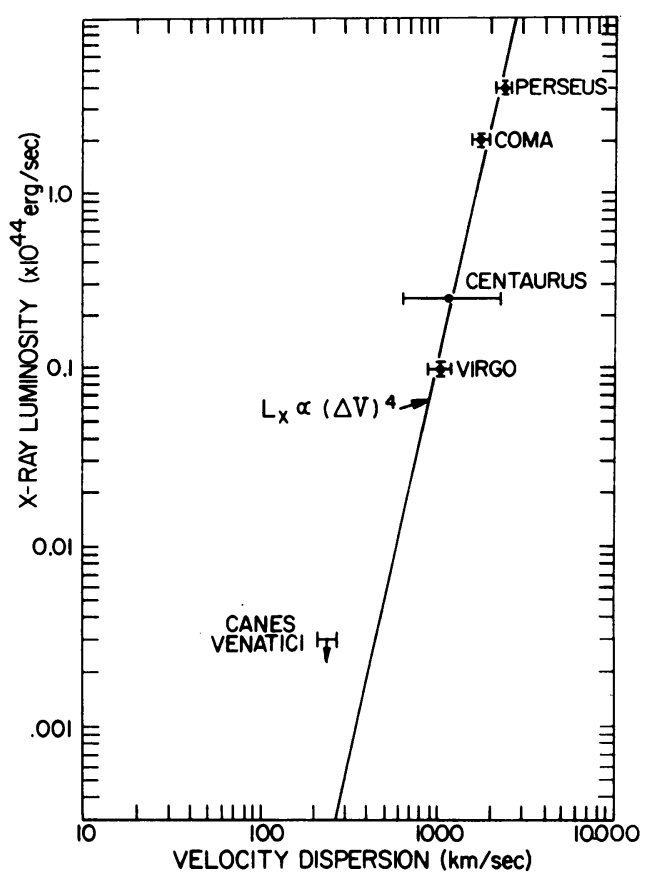

Fig. 6. Luminosity versus velocity dispersion for X-ray sources in clusters. The curve is arbitrarily normalized. For the three clusters with well determined velocity dispersions, the correlation between $\Delta V$ and $L_{x}$ is undeniable. More detailed analysis may prove that the data fit a curve slightly different from the $(\Delta V)^{4}$ curve shown, however. (Solinger and Tucker, 1972).

in Figure 6. This effect was noticed by Solinger and Tucker (1972). The observed luminosities are consistent with a fourth power dependence on $\Delta V$. If this type of correlation holds up under future observations, we must interpret that there is a connection between X-ray emission and cluster dynamics involving the presence of some fraction of the virial mass in intergalactic gas, and that thermal bremsstrahlung is the X-ray emission mechanism. This would be the first unambiguous observation of intergalactic gas, whose presence has been suspected in the past, but not confirmed.

Some new UHURU observations of the spectra of extragalactic sources are shown in Figure 7. The ordinates are logarithmic and represent relative counting rates. The abscissae represent photon energy. In the upper right corner, is the spectrum of the Crab Nebula, which we use as a standard. We assumed interstellar absorption corresponding to $(1.6 \pm 0.16) \times 10^{21} \mathrm{H}$ atoms $/ \mathrm{cm}^{2}$ as observed in radio, which corresponds to $E_{a}=0.45 \pm 0.02 \mathrm{keV}$. The spectral index was allowed to vary, with the best fit value being $0.99 \pm 0.05$. The histogram for the Crab represents both the observed count rate distribution in the UHURU pulse height analyzer and the calculated fit, because the statistical errors are very small. The middle column shows the count rate distributions from cluster sources as histograms with $1 \sigma$ error bars. The circles are the computed fits. Perseus - NGC 1275 is similar to the Crab which has a power law index of 1 . 

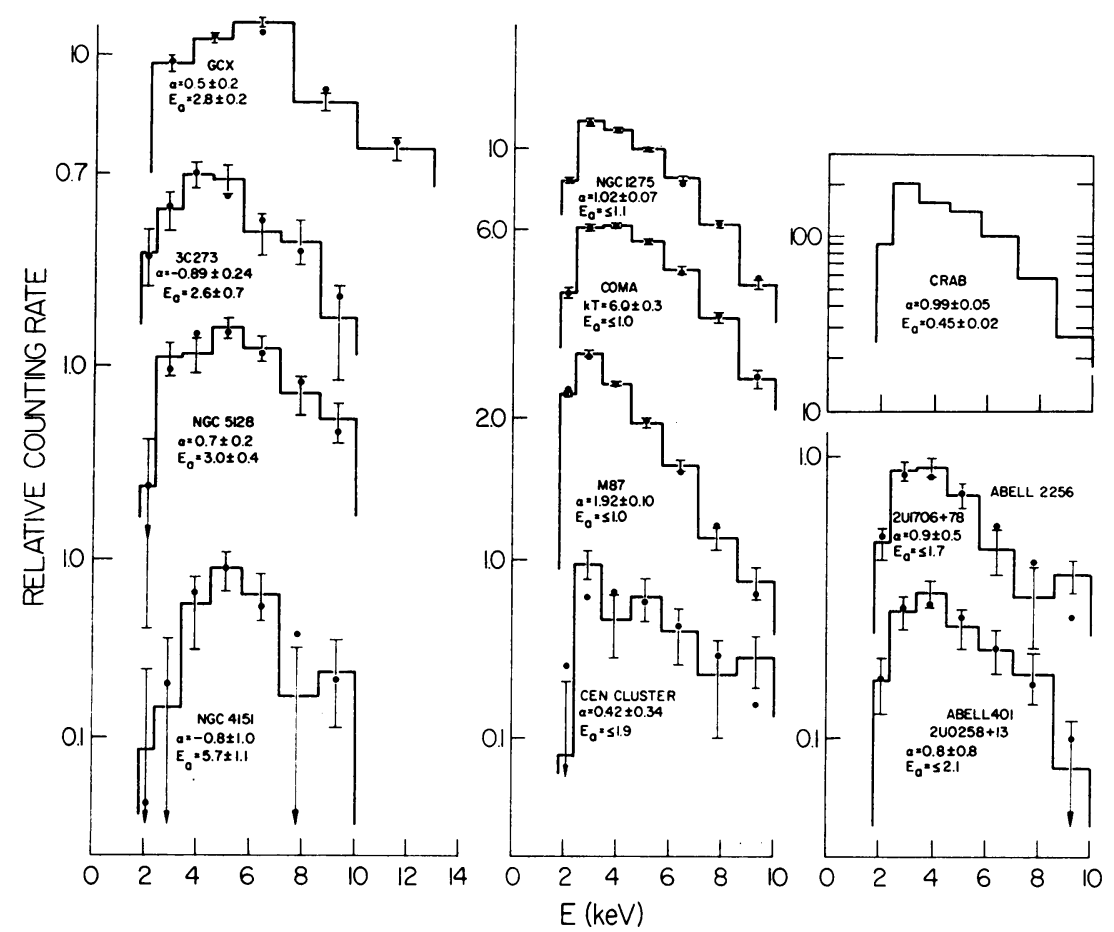

Fig. 7. Spectra of extragalactic sources. Counting rates versus energy are plotted as histograms with one sigma error bars. The closed circles are the predicted count rates assuming model spectra as inputs and computing the count rate after accounting for counter efficiency, pulse height resolution and fluorescence escape effects. The slope is given as $\alpha$ for the energy index of a fit to a power law, or $k T$ for an exponential. $E_{a}$ is the low energy cutoff in $\mathrm{keV}$, assuming photoelectric absorption. The four spectra in the left column show a drastic turnover at low energies. The spectra in the center column and the two lower ones in the right column are from sources located in clusters, and show no cutoff. The spectrum of the Crab Nebula is shown at the top of the right column for comparison.

M 87 has a steep spectrum with a power law index close to two. The spectrum of the Coma cluster fits better to isothermal bremsstrahlung than a power law; $k T$ is $6.0 \pm 0.3 \mathrm{keV}$. The spectra of the Cen cluster and Abell 2256 and 401 are similar to Coma and NGC 1275 in that they are significantly flatter than that of $M 87$. We see no definite low energy cutoff in any cluster source.

The left column shows data from individual galaxies and $3 \mathrm{C} 273$. They all have a cutoff. The top spectrum is from the source at the nucleus of our own galaxy, GCX (Kellogget al., 1971b). The suggestion is that we are seeing X-rays from the nuclei of these other galaxies as well. Since we see no cutoffs in the cluster sources, we are led to believe that the X-ray emission mechanism is different for clusters than for individual galaxies. In particular, it appears that the cluster sources are truly diffuse, and not just a collection of active galaxies.

As an example of how we can use these observed cutoffs, consider 3C 273. We find a cutoff of $2.6 \mathrm{keV}$. This is probably not due to intergalactic material since such mate- 
rial at the critical density would not have the heavy element abundance required to give that much absorption; also it must be very hot if present, so it would be ionized and absorb still less. The absorption is most likely at the source, and corresponds to $5 \times 10^{22}$ atoms $/ \mathrm{cm}^{2}$ assuming normal galactic abundance ratios in $3 \mathrm{C} 273$.

If we assume that the $\mathrm{X}$-rays and the point radio source $3 \mathrm{C}$ 273B are coming from the nucleus of a galaxy, then the lack of a radio cutoff down to $\cong 80 \mathrm{MHz}$ means that the cloud of material surrounding the source has a radius $>30 \mathrm{kpc}$ along the line of sight, assuming a temperature of $10^{4} \mathrm{~K}$. This, in turn, places $3 \mathrm{C} 273$ more than $30 \mathrm{kpc}$ from the Sun.

Figure 8 shows NGC 5128 or Cen A. This is a giant radio galaxy with two lobes several degrees in size. There are also two inner lobes just $7^{\prime}$ apart centered on the optical galaxy. The galactic nucleus apparently has been observed as an IR hot spot (Kunkel and Bradt, 1971 and Becklin et al., 1971) and a compact radio source (Wade et al., 1971). We have found that the $\mathrm{X}$-rays are coming from a source $\leqslant 10^{\prime}$ in size whose location is centered on the optical galaxy. Our upper limit on X-rays from the

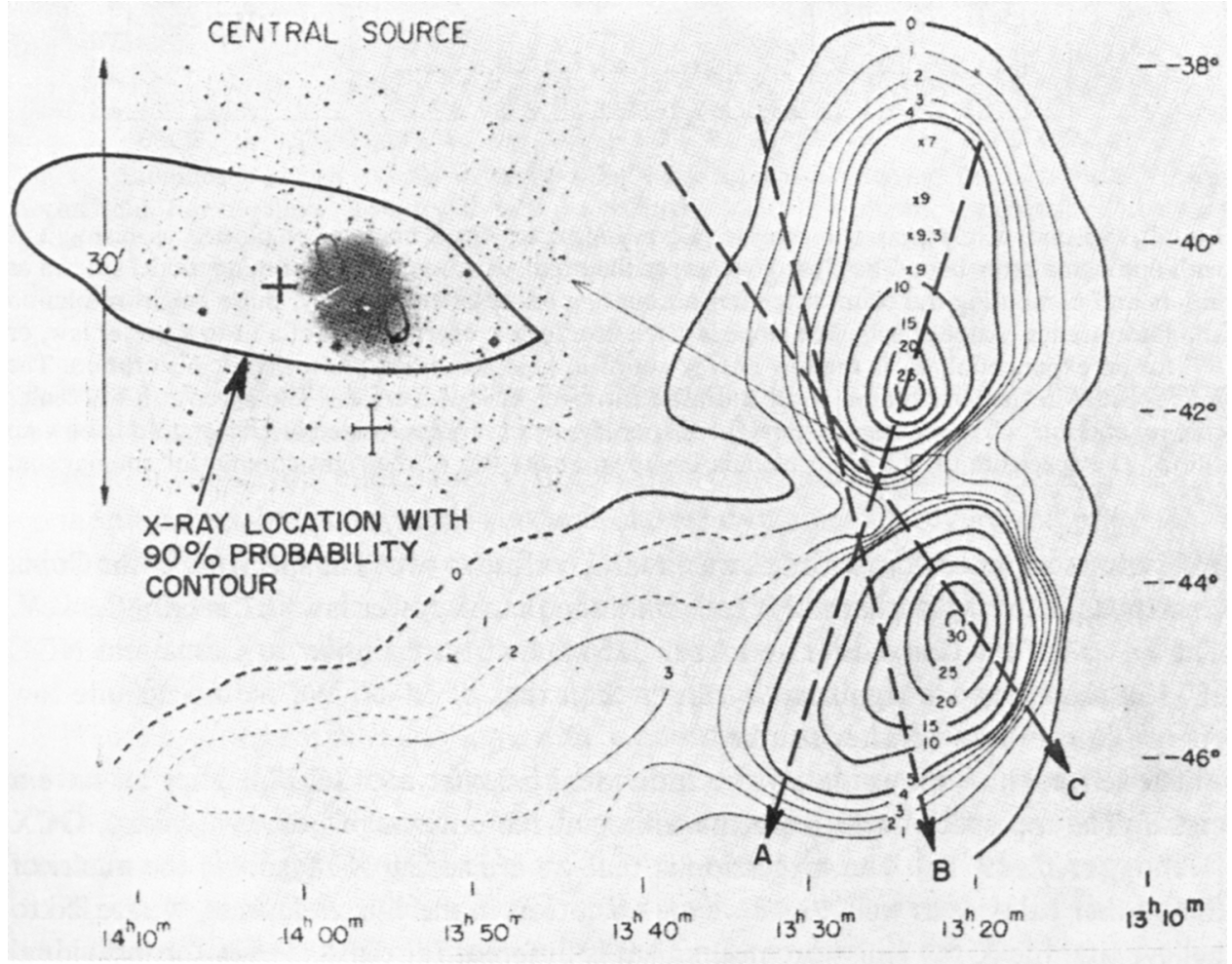

Fig. 8. Centaurus A. The optical galaxy NGC 5128 is located within the X-ray source error box. The size of the source is less than $10^{\prime}$. This still allows the X-ray emission to be coming from the inner radio lobes shown in the inset, or from the optical galaxy. The nucleus of the optical galaxy is the most likely location of the source, since it is so strongly cut off at low X-ray energies, due to absorption. 
outer lobes sets an upper limit on the temperature of the universal blackbody radiation of $5.1 \mathrm{~K}$, assuming a magnetic field strength of $4 \times 10^{-6} \mathrm{G}$ in the lobes which corresponds to equipartition between magnetic and electron energy density. Or, if we fix the blackbody radiation at $2.7 \mathrm{~K}$, we obtain a lower limit of $1 \times 10^{-6} \mathrm{G}$ in the giant lobes of Cen A (Tucker et al., 1972). The X-ray source is probably coming from the nucleus of NGC 5128, since we see it strongly cut off. This must be the center of the high energy activity which has produced the lobes in the past, but until recently has been obscured from us by the great amount of dust in NGC 5128.

The unidentified high latitude sources are listed in Figure 9. The first group are located near $3 \mathrm{C}$ or $\mathrm{MSH}$ radio sources. The second group are not associated with strong radio sources. The six coincidences between $\mathrm{X}$-ray sources and strong radio

1. NEAR STRONG RADIO SOURCES ( $\geq 30$ F.U.)

$\begin{array}{ll}\frac{2 U}{\text { RADIO }} \\ 0410+10 & \text { 3C113 (WEAK) } \\ 0515-34 & \text { MSH 05 }-310 \\ 0525-38 & \text { MSH 05 }-36 \\ 0426-63 & \text { MSH 04 }-64 \\ 1253-28 & \text { MSH } 12-212 \\ 2128+81 & 3 \text { C435.1 }\end{array}$

2. UNCERTAIN I.D.

$\begin{array}{ll}\frac{2 U}{21} & \text { CANDIDAIE } \\ 0043+32 & \text { ZW O041.1+3235 OR OB + 368 } \\ 0426-10 & \text { (SOUTH) } \\ 0440+7 & \text { CLUSTER? } \\ 0544-39 & \text { (SOUTH) } \\ 0628-54 & \text { (SOUTH) } \\ 1231+7 & \text { IC 3576? } \\ 1420-02 & \text { NGC 5604 } \\ 1443+43 & \text { CLUSTER? } \\ 1808+50 & \text { ABELL 2298? } \\ 1843+67 & \text { CLUSTER? } \\ 1849-77 & \text { (SOUTH) } \\ 1954-68 & \text { (SOUTH) } \\ 2134+11 & \text { CLUSTER? } \\ 2346-32 & \text { NGC 7793? } \\ 2358-29 & \text { (SOUTH) }\end{array}$

Fig. 9. Unidentified high latitude X-ray sources $\left(|b|>20^{\circ}\right)$.

sources are somewhat higher than would be expected on a chance basis and may be due to a real physical association. Among the others there are probably many chance coincidences, such as the three sources near undistinguished NGC or IC galaxies.

The latitude distribution of these sources is shown in Figure 10. This has been corrected for nonuniform sky coverage. The horizontal line is the mean for all latitudes above $20^{\circ}$ and the dashed lines are $\pm 1 \sigma$. The apparent excess in the 20 to $43^{\circ}$ zone is not statistically significant, being only $1.4 \sigma$. Therefore, to the best of our knowledge, they are distributed uniformly on the sky. Suppose these sources were very close to us, 
within the disk of our galaxy. Then we would have observed the integrated effect of other sources similar to them but further away as a galactic ridge of X-ray intensity. Such a strong ridge has not been seen. Therefore, we believe these unidentified sources are a new class of distant extragalactic object.

Figure 11 shows the luminosity function of high and low latitude sources (Matilsky et al., 1972). The low latitude sources were discussed by Tananbaum (1972). The high

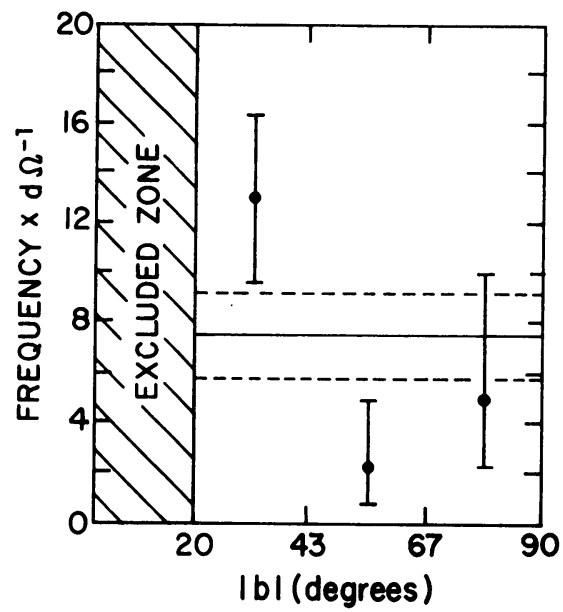

Fig. 10. Galactic latitude distribution of unidentified X-ray sources. This is a plot of the distribution of the high latitude sources listed in Figure 9, with galactic latitude. Corrections have been made for the solid angle, sky coverage in each zone and sensitivity versus source strength. The solid horizontal line is the mean, and the dashed lines are one sigma errors in the mean.

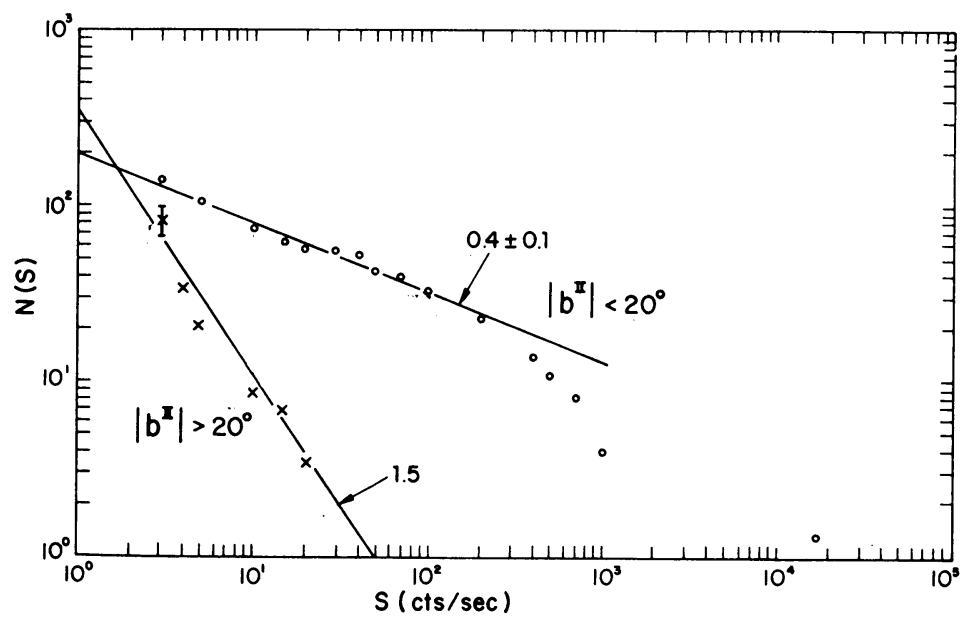

Fig. 11. Integral distribution of number of sources versus strength, $S$. The sources are those given in the UHURU Catalog; the numbers given have been corrected to complete sky coverage and to $100 \%$ source detection efficiency down to the limit of 3 counts $\mathrm{s}^{-1}$. The high latitude sources are consistent with a distribution of constant luminosity sources distributed uniformly throughout space, giving a distribution with a slope of 1.5 as shown by the solid curve drawn through the X's. 
latitude sources agree well with a 1.5 power law expected for a uniform volume distribution, with constant space density and luminosity.

Does the luminosity function continue to weaker sources than we have yet observed? Figure 12 shows the fluctuations in the background counting rate obtained as differences in adjacent $5^{\circ}$ regions in the sky. The curve is that expected from counting statistics. The excesses at 1.6 to 2.4 counts $\mathrm{s}^{-1}$ are most likely due to sources below our present threshold of detectability. The estimated number at this intensity level fits well on the $\log \mathrm{N} \log \mathrm{S}$ curve, which predicts that we will observe about 300 high latitude sources down to 1 count $\mathrm{s}^{-1}$ with UHURU eventually.

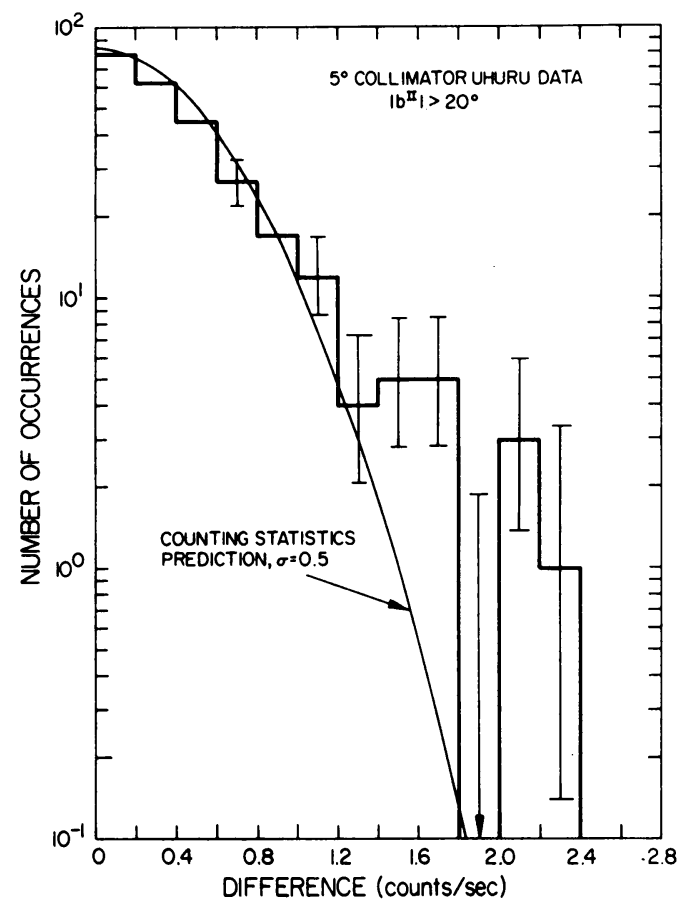

Fig. 12. Point-to-point Count Rate Differences. Data from each great circle scan at high latitude are analyzed for fluctuations by taking the difference in absolute magnitude of count rate between adjacent $5^{\circ}$ bins. The frequency distribution of these differences is shown here. If the differences were due only to counting statistics, we would expect the solid gaussian curve. We observe excesses at about

1.6 counts $\mathrm{s}^{-1}$ and 2.2 counts $\mathrm{s}^{-1}$. These are undoubtedly due to weak sources just below the UHURU threshold of detectability.

With this positive indication that still weaker sources than we have yet observed exist, we can integrate the contributions of the various types of sources to the X-ray background. Figure 13 shows the estimated contributions from identified classes of $\mathrm{X}$-ray sources. About equal contributions are obtained from Seyferts and clusters. We assume that one third of all Seyferts are X-ray sources, based on our results for 


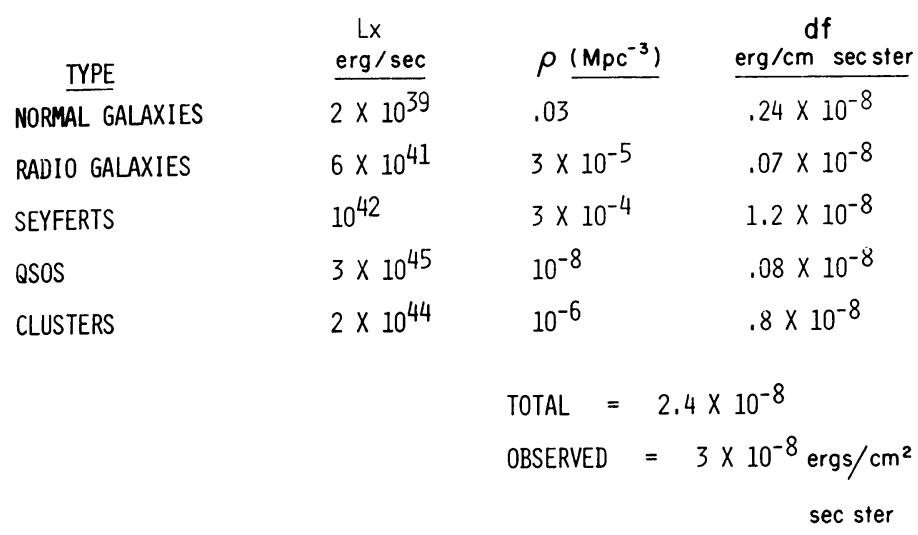

Fig. 13. Contributions to the diffuse X-ray background (2-6 keV). The two types of source which could contribute significantly to the background are Seyferts and clusters.

NGC 4151, 4051 and 1068. The contribution from Seyferts could be much less than we have calculated if the fraction of Seyferts which are strong X-ray sources like NGC 4151 is much less than $\frac{1}{3}$.

We could approach the problem differently by integrating the $\log \mathrm{N} \log \mathrm{S}$ curve out to a faint source limit of $5 \times 10^{-3}$ counts $\mathrm{s}^{-1}$ to explain the background. If we assume that this intensity cutoff corresponds to the edge of the observable universe, we have a distance scale for the twenty or so weak sources already detected at about 3 counts $\mathrm{s}^{-1}$. They are about $150 \mathrm{Mpc}$ away, perhaps not close enough to have been noticed in earlier surveys in optical and radio. It seems that a large fraction of the allsky X-ray background is certainly contributed by distant rich clusters of galaxies and possibly Seyferts. The rest may be contributed by the unidentified class of sources extrapolated to great distances.

It is to be expected that further study of the powerful extended X-ray sources in clusters and galaxies will tell us much about the evolution of clusters and perhaps about cosmological phenomena. Based on the results presented here, one may speculate that the Virgo and Perseus clusters are similar in that they contain an active galaxy on which an extended X-ray source is centered; the source has a power law spectrum. Also, these two clusters are irregular. In Coma, we do not see such a prominent active galaxy. The source may be centered on the kinematic center of the cluster, and seems to show a spectrum closer to that of isothermal bremsstrahlung at a temperature corresponding to the RMS velocity of galaxies in the cluster. Also, the cluster has a more symmetrical appearance, and is believed by many to be bound. If the X-ray emission from these clusters is due to hot gas, we may be seeing the active galaxies in Virgo and Perseus heating up the gas contemporaneously, whereas in Coma we see the thermalized remnant of a heating process which ended more than $10^{9} \mathrm{yr}$ ago.

The rich clusters, being very luminous and of great size, will be observable with finite angular size out to distances where redshift effects dominate. At $3200 \mathrm{Mpc}$, with 
$Z=1.4$, sources like the Coma cluster will have an angular size of one arc minute and should be observable with large orbiting telescopes of the near future. We may even be able to detect clusters out to $Z=3$, and learn about evolution of clusters in the early stages of the formation of the universe.

\section{Acknowledgements}

I wish to acknowledge the assistance of all the members of the UHURU group at AS \& E, in the preparations for this paper, especially Stephen Murray, Terry Matilsky, Herbert Gursky and Riccardo Giacconi; also William Forman of the Harvard College Observatory.

\section{References}

Becklin, E., Frogel, J., Kleinmann, D., Neugebauer, G., Ney, E., and Strecker, D.: 1971, Astrophys. J. Letters 170, L15.

Bowyer, C., Lampton, M., Mack, J., and deMendonca, F.: 1970, Astrophys. J. Letters 161, L1.

Bradt, H., Mayer, W., Naranan, S., Rappaport, S., and Spada, G.: 1967, Astrophys. J. Letters 161, L1.

Byram, E. T., Chubb, T. A., and Friedman, H.: 1966, Science 152, 66.

Forman, W., Kellogg, E., Gursky, H., Tananbaum, H., and Giacconi, R.: 1972, to be published.

Fritz, G., Davidsen, A., Meekins, J., and Friedman, H.: 1971, Astrophys. J. Letters 164, L81.

Giacconi, R., Murray, S., Gursky, H., Kellogg, E., Schreier, E., and Tananbaum, H.: 1972, Astrophys. $J$. (Dec., to be published).

Gursky, H., Kellogg, E., Murray, S., Leong, C., Tananbaum, H., and Giacconi, R.: 1971, Astrophys. J. Letters 167, L81.

Gursky, H., Solinger, A., Kellogg, E., Murray, S., Tananbaum, H., Giacconi, R., and Cavaliere, A.: 1972, Astrophys. J. Letters 173, L99.

Kellogg, E., Gursky, H., Leong, C., Schreier, E., Tananbaum, H., and Giacconi, R.: 1971a, Astrophys. J. Letters 165, L49.

Kellogg, E., Gursky, H., Murray, S., Tananbaum, H., and Giacconi, R.: 1971b, Astrophys. J. Letters 169, L99.

Kellogg, E., Gursky, H., Tananbaum, H., Giacconi, R., and Pounds, K.: 1972, Astrophys. J. Letters 174, L65.

Kunkel, W. and Bradt, H.: 1971, Astrophys. J. Letters 170, 27.

Leong, C., Kellogg, E., Gursky, H., Tananbaum, H., and Giacconi, R.: 1971, Astrophys. J. Letters 170, L67.

Mark, H., Price, R., Rodrigues, R., Seward, F., and Swift, C.: 1969, Astrophys. J. Letters 155, L143.

Matilsky, T., Gursky, H., Kellogg, E., Tananbaum, H., Murray, S., and Giacconi, R.: 1972, Astrophys. J. Letters, to be published.

Meekins, J., Gilbert, F., Chubb, T., Friedman, H., and Henry, R.: 1971, Nature 231, 107.

Schreier, E., Giacconi, R., Gursky, H., Kellogg, E., and Tananbaum, H.: 1972, Astrophys. J. Letters, to be published.

Solinger, A and Tucker, W.: 1972, Astrophys. J. Letters 175, L107.

Tananbaum, H.: 1972, this volume, p. 9.

Tucker, W., Kellogg, E., Gursky, H., Giacconi, R., and Tananbaum, H.: 1972, Astrophys. J., to be published.

Wade, C., Hjellming, R., Kellermann, K., and Wardle, J.: 1971, Astrophys. J. Letters 170, L11. 